\title{
Bodily Awareness: An Exploration in Critical Design
}

\author{
Daniel Brynolf, Vanessa Carpenter, Mads Hobye, Henrik Svarrer Larsen \\ Masters, Interaction Design \\ Malmo Hogskola, K3 \\ vanessajcarpenter@gmail.com
}

\begin{abstract}
There exists a routine in most people's lives wherein they wake, work, and sleep with few activities in between those main moments. During this routine, typical behaviors occur wherein the participants are completely numb to the activities that they are completing each day, they are so used to doing them that they no longer are aware of their movements. Work and everyday life is so typical that people feel the need to exercise to offset their lack of movement, their typical actions. Assuming this situation, we seek to explore how everyday bodily attention and movement can be challenged, and made aware. If there is a possibility that we can make people aware of their full body movements, we can therefore create the tendency for people to become more explorative in their daily lives. We explore concepts of the body-mind disconnect, introduce elements of curiosity and examine unawareness of body.
\end{abstract}

We developed a process of research wherein we conducted initial research observations, a series of low, mid and high fi prototypes, body movement workshops, and cultural probes to examine how people experience bodily awareness.

\section{Categories and Subject Descriptors}

I.4.8 [Image Processing and Computer Vision]: Scene Analysis; F.1.2 [Modes of Computation]: Interactive and Reactive Computation

\section{INITIAL OBSERVATIONS OF BODY MOVEMENT}

We conducted initial observations of bodily movement by going to several fitness centres. Here we observed how the mind body disconnect is prominent in the fitness centre. We learned that people within fitness centres tend to separate place and time and body and mind. People would listen to music to distract themselves from their surroundings or possibly from their physical exertion; they would read magazines on the cardio equipment further separating

(C) The Author 2008

Published by the British Computer Society where they were to what they were doing. The machines in the gym worked body parts separately, separating the body in segments rather than treating it as a whole. From these concepts, we determined that we might be able to raise people's bodily awareness in their everyday activities, such as walking, typing, or getting a coffee, through engaging their full body during movement, through integrating the body and mind, and through examining social expression, what is the normal social body movement that is acceptable?

\subsection{Low-Fi Prototype: Body Workshops}

Following this, we conducted a series of body workshops and low-fi prototypes to examine how people reacted to interferences with their typical body movement. Our first prototype consisted of creating a 'Wizard of Oz' effect wherein we would make noises in sync with people's body movements when they walked by. We conducted several bodily motion exercises whereby we tried to become more aware of certain body areas. We imitated each other's methods of walking to try to become more aware of our own walking patterns, and did an exercise wherein we would touch a point on another team member, and then be 'attached' to them by that point as they moved around. This made the team member aware of that point to a great degree, and aware of what effect the movements they made with it might have. There needed to be an extremely tight coupling between movement and sound to be convincing.

\subsection{Low-Fi Prototype: Sounds when stepping}

We created another low-fi prototype which consisted of contact microphones and a 'plop' sound which would sound each time someone stepped on the floor. This sound was much more tightly coupled to movement, and as people walked by, their footsteps were essentially amplified to become a different noise, playing out as they walked by. People noticed this and some played with the system, dancing, tapping, and trying to walk quickly or slowly to see the effects. In one instance, one person even removed their shoes to walk across the area so that they could avoid making noise.

\subsection{Low-Fi Prototype: Bells}

The third low-fi prototype we tried consisted of pinning bells on to test subjects, who wore the bells on their shoulders, belt, and knees. They wore them for an entire day and reported back to us when they noticed movements that they otherwise would have ignored, typical tendencies that they performed, such as brushing hair back with hand, and when they stood up or sat down. 


\subsection{Results of Low-Fi Prototypes}

In each of these low-fi prototypes we learned an important aspect of how people are or are not aware of their bodily movements. With the body workshops, we all became more aware of our own body movements and were suddenly focused on a particular body part, able to discern its movement from the rest of our typical movements. The Wizard of $\mathrm{Oz}$ prototype indicated to us that there needed to be a very strong association between sound and movement.

In each of these situations, it must be noted that initial tests were done at our school in which people are used to seeing interactive installations and do not react quite as easily to strange occurrences, as might someone who is unfamiliar with such installations. We decided that to test the low fi prototypes more precisely we should test them out within the context of an office building, where our main focus of people who did similar tasks and movements each day would be present.

\section{CONTEXT: OFFICE ENVIRONMENT}

In the office environment the contact microphones were set up to record steps on a main staircase in the middle of many offices. Many employees used this staircase, and had to take clients up and down the stairs at regular intervals. The prototype worked well in this situation as the stairs provided a distinct sound each time someone took a step. It was observed that people would run up the stairs, take two stairs at a time, or even take a different staircase once they realized they were creating the resulting sound. When people first encountered the sound, they either didn't notice, or were surprised. One person even tapped on the stairs several times to assure that he was making the sound. People seemed interested and body movement was definitely changed by the presence of the sound. People changed their normal behaviors, which had been observed before prototyping, to either avoid or play with the sound when the prototyping was active.

\section{MID-FI PROTOTYPE: WEBCAM TEST}

After completing many low-fi prototypes we moved on to the mid-fi prototype, a collection of tests based on a webcam detecting movement. This is the beginnings of an exploration in 'Computer Vision', using a webcam as an input device to detect movement or track colours. We wanted to create an invisible area that would react to people's movements, much like the 'Wizard of Oz' tracking people's body movements and the contact microphones picking up steps, this was a combination, a focus on whole body movement in space.

\subsection{Objects in space}

The first webcam test created certain areas in the webcam's field of vision that would play a sound if movement was detected in those areas. In this test, participants were able to walk into a room or an area, and be interacting with the specific areas on the webcam screen instantly. Simply by walking through the field of vision, participants activated the sounds, and upon experimentation, would learn where the areas of sound were located, and were able to manipulate the air around them, activating different spots in various sequences. This allowed the participant to move their bodies freely through space in order to reach the goal of activating sounds. As explained in Hertzian Tales, "Electronic objects are disembodied machines with extended invisible skins everwhere. They couple and decouple with our bodies without us knowing." [1] Our webcam prototype explored this concept, giving people a disembodied machine in the form of trigger points in the air to explore. When people activated the triggers, they were 'coupling' with the technology, and when they understood the trigger points, they were able to choose whether or couple or decouple with the system. There was, however, not a concrete connection between movement and sound as participants could not determine what caused the sound they made, and as such, it was difficult to determine whether their interaction was deliberate or coincidental.

\subsection{Object and Colour Tracking}

To improve the connection between movement and sound, we changed the webcam program to instead track objects, and then eventually colour. When tracking objects, the program could be told to choose an object, such as a person's head and then track that, reacting with varying sounds depending on how much the object moved. Since this required us to physically click on an object within the webcam's field of vision, we decided to instead track colour. This seemed to create a better connection between movement and sound. By interfacing with colours and movement, we reduced the need to depend on technology, "the challenge today is not to create electronic space, but eletronic-free space." [2] We felt that this method suitably reduced the electronics and the awareness of the presence of technology in the prototyping, which allowed participants to simply experience the space as it was, through colours and movement rather than interface.

\section{HIGH-FI PROTOTYPES}

\section{- FINAL DESIGNS}

After many different prototyping sessions and running cultural probes, we had learned a lot about bodily movement, and people's awareness of their own bodies. We understood primarily that people associated disturbances in their everyday routines with their environment. There was a definitive lack of association between what was happening and people's body movements, instead they simply focused on what sounds were occurring in their environment, and how their environment was affected. This was most noticeable during the webcam tests. People didn't associate their movements to sounds immediately and were instead focused on the speakers or the room, trying to investigate where the sound was coming from. Again, it became apparently that a tight coupling to sound was necessary to create the body movement - sound connection. We refocused our concepts and created two final prototypes for testing, the Harmony Staircase and the Bodily Awareness Device.

\section{HARMONY STAIRCASE}

The Harmony staircase was an iteration of the contact microphones in the office context. We wanted to again focus on the staircase as it was a good indicator to participants of their body movement, they were easily able to draw a connection between their steps on the stairs to the sounds they were hearing. Our solution was to have a webcam mounted above the stairs, when people went up or down the stairs, the webcam would track their movement, and play a harmony of chords, ascending or descending in relation to their movement. In this way, people could hear a progression of 
sounds as they moved. Speakers and other devices were hidden from view so as people came up and down the stairs, it was a completely surprising experience for them, unless they had seen us setting up the equipment initially. When interviewed, people said that they were intrigued by the sound, but that they could not draw a correlation between their movement up and down the stairs and the sounds they were hearing. Again, we realized that people were still more focused on their environment than their bodily movement.

\section{BODILY AWARENESS DEVICE}

A prototype was created to determine if a personal experience with sound and body movement would be beneficial. Within this concept, participants could wear a box that would give an indication of body movement. A fundamental aspect of this concept was the use of a single-sided headphone, participants were still able to hear their environment but were also given a direct feed of sounds created by their body movement. This established to participants that the sounds they heard were a result of their body movement, and not of their environment. Further, since people would have to carry the device with them, which is both unnatural and a burden, they were forced to be aware of their body movements because they had a constant reminder of to pay attention to body movement.

\subsection{Design of Bodily Awareness Device}

The bodily awareness device consisted of a Wii-Nunchuck connected to an Arduino board, with a single-sided headphone providing sound output. The Nunchuck was either held in hand, or attached to the body to gather $\mathrm{x}, \mathrm{y}$, and $\mathrm{z}$ movements. These movements would be read by the Arduino board which would then output corresponding sounds in relation to the position of the Nunchuck. The entire device was encased in a small box with a LED to provide visual feedback of read movement.

\subsection{Testing the Bodily Awareness Device}

Participants felt a strong connection between body movement and sound. They easily recognized that their body movement was triggering sound and that the more they moved and in different ways, would result in new sounds. They were curious to explore these sounds and were comfortable moving in many ways and exaggerating their movements to understand how they could affect the sound. Unfortunately, the sound was very restricted, since the Arduino board can only output a limit range of sound. As such, the sounds were not necessarily the best choices we could have made given an extensive sound library, but working with the range of sounds available, we calibrated the device to output different tones based on movement.

\section{DEVELOPMENT OF INTERVIEWS}

Questions were developed for two groups, those were passing by the installation and might interact with it within the context of our school, and those in the office environment who were participating simply by carrying out their everyday duties and activities in their workplace. These two sets of questions helped us to understand people's reactions to the installation, and to whether or not they had been made more aware of their bodily movement as a result of interacting with it.

\subsection{Outcomes of Interviews}

Participants in the sound-stepping prototype could be divided into two groups, those were engaged with the system by moving through the environment and those were were affected by the sound and were not participating in it. People who participated in it indicated that they were intrigued by the sound but hadn't necessarily understood that it was them causing the sounds. People who spent more time with the system began to play with it, trying to challenge the sounds by running, hopping, and dancing across the area. Different types of footfalls and shoes were tried, and distance of interaction, how far away a person could be and still activate the system, was tested using different types of shoes, for example, high heels which had a very strong effect with the system.

Participants in the webcam tests didn't fully understand what they were experiencing. They understood that as they moved through the space, sounds were playing but couldn't understand that there were specific trigger points in the air or that colour or motion dictated what sound would be played. For the most part, participants were simply trying to walk from one area to another and had not entered the space with the intention to engage the system. One participant expressed that she was uncomfortable with the system, and didn't want to draw attention to herself. She likened the experience to wearing high heels in an echoing area, where people look to the source of the noise and stated that in that situation, she would walk on her tip toes to avoid the sound, so the fact that our prototype purposely made her a 'performer' [4] was not a positive experience for her. However, since she did change her body movement by walking on tip toes, or by rushing through the area of interaction, it was apparent that the prototype made her more aware of her body and her body movement.

Participants of the Harmony Staircase (in the context of the office environment) were in general, intrigued by the sounds. They had not for the most part, noticed any of the equipment and as such, simply listened to the sounds of the staircase as they moved up and down it. Participants stated that there were both positive and negative reasons to have this installation in a workplace, that it was interesting and generated discussion, but that over time, the sound became a distraction for those working around it. Several participants reported that they thought the music was louder when they were descending the stairs. They did not report any associations between body movement or stair climbing with activating the music. They simply understood that using the staircase somehow activated the sounds.

Participants using the Bodily Awareness Device indicated that they did not feel natural carrying around or wearing the device but that they did have a strong association between their movement and the corresponding noise. They stated that they did not feel that the sounds were appropriate to represent body movement but they were fascinated to play with the device and experiment with exaggerated body movements to activate the sounds.

\section{REFLECTIONS}

This project encompassed a range of research into theories regarding body movement, body awareness, the mind body 
connection, and critical design. It was essential for us to carefully analyze each prototyping session we did to learn as much as possible about if we can cause people to become more aware of their body movements through simple 'distrubances'. Lepecki, quoting Foucault discussed "constructions of resistence" [4], which we try to utilize in all of our prototypes to create an effect. We created small disturbances in people's everyday lives, whether it was causing a noise to sound when they took a step or swung their arms, or when we made an invisible field of triggers using the webcam to catch their movements so as they innocently walked through an open space, they activated sounds. These constructions of resistance aided us in understanding people's reactions to their environments, that they would notice a change in environment and sound, but would or could not necessarily associate this to body movement.

When people engaged with our system, they were performing, although it was not always their choice to do so, as they made sound, people around them would glance up and watch their movements. We were projecting performance on to people who hadn't even intended to participate in the first place. This made some people uncomfortable and caused them to leave the area quickly, and made other people feel like they could perform more readily, encouraging them to dance and move in playful ways. We found it interesting that people depended on a social setting to "defines which movements that are appropriate to use for interaction". [5] They would move based on how comfortable they were in a given social setting, and had varying levels of comfort with movement based interaction.

Since in the first rounds of prototyping, until the Harmony Staircase and the Bodily Awareness Device, we were focused on the free movement of people, it was important to us to "preserve the spontaneity as well as ambiguity in human movement". [5] We didn't want to establish specific movement patterns or push people to have predefined movement patterns that they must perform to gain a response. Moen explains that usually this is the case for successful interaction, and as a result, we lose the spontaneity and ambiguity present. By not requiring people to perform specific actions we had hoped to give them freedom to act in any way, to be spontaneous, to take part in the system. However, it was quickly made aware to us that this would not be possible, people simply did not participate when they were not aware of the situation, whether due to social discomfort or simply by not even noticing that they were interacting with a system.

It is these combinations of subtle change, of small disturbances in everyday life, leading up to the very prominent presence of the Bodily Awareness Device, that convince us of the validity of "transformation design". [3] This design convinces us that through small changes in every day life, larger effects can happen. Perhaps because we made a staircase Harmonic, people will take the stairs more quickly or slowly, or more often, perhaps in hopes to hear the sound once more. Perhaps the bodily awareness device helps people to be more spontaneous over time, and to be more playful in their movement, so much so, that when the device is no longer present, they continue, in some small but significant manner, to pay attention to how they move, and what they do in their given daily context.

It is our hope that everyone who has encountered our prototypes has a better understanding and awareness of their body movement, that they can at some point go through the same hallway and recall how they were made aware of their body movement, and that only a few seconds of thought can bring them back to that awareness. The possibilities for further research are many, and it would be very beneficial to see if this type of small intervention could lead to a larger social change wherein people feel free to move in exaggerated ways, where they happily had more 'bounce' in their step and where their physical exertion in the office context was not simply reaching for their mouse, but rather walking or taking the stairs in some new creative manner that helped them to connect body as a whole, and body and mind together.

\subsection{Future Work and Considerations}

It would be very interesting to continue this study on a larger scale, including different types of body movement sensors, such as accelerometers. We began to explore this through the use of the Wii Nunchuck, and would be interested to see if a completely integrated system could be created whereby through the use of computer vision and the invisibility of electronics, we could create a system which motivated people to be aware of their body movement, and to extend and change the way they moved their bodies, that would be our ultimate goal. We feel that a huge potential exists to make an awareness of body movement and that people simply haven't got the time, energy, or interest to explore this in their everyday lives, but that an exploration in body awareness and movement would be very beneficial for anyone in the fast-paced, technology-dependent world that we live in today. A disturbance created in people's everyday routines is our goal, to change something subtly that will, over time have an effect in changing the lives of those involved.

\section{ACKNOWLEDGMENTS}

\subsection{MINC - Incubator}

Tack sa mycket to the staff of MINC who put up with a lot of annoying noises from our prototypes.

\subsection{Half Machine}

Thank you to Half Machine for use of their equipment and venue.

\section{REFERENCES}

[1] A. Dunne. Hertzian Tales: electronic products, aesthetic experience, and critical design. 2005.

[2] R. F. Dunne, A. Design Noir: The Secret Life of Electronic Objects. 2001.

[3] A. Emilsson. Presentation: Critical Design. 2008.

[4] S. Lepecki, A. Banes. Introduction: The Performance of the Senses. 2007.

[5] J. Moen. From hand-held to body-worn: embodied experiences of the design and use of a wearable movement-based interaction concept. 2007. 